\title{
Evolution de la photosynthèse du peuplier au cours d'un cycle d'infection par Marssonina brunnea : comparaison de 3 clones
}

\author{
Patricia MAURER *, E. DREYER **, et J. PINON * \\ avec la collaboration technique de P. GROSS \\ INRA-Nancy, B.P. 35, Champenoux, F 54280 Seichamps \\ * Laboratoire de Pathologie forestière \\ ** Laboratoire de Bioclimatologie forestière (Station de Sylviculture \& Production)
}

\begin{abstract}
Résumé
La réduction de photosynthèse d'une feuille de peuplier, consécutive à l'infection par Marssonina brunnea (Ell. \& Ev.) Magn., champignon parasite foliaire, se manifeste de deux façons distinctes lors du développement de la maladie : pendant la période d'incubation elle est brutable et partiellement réversible, puis elle se poursuit de manière lente et progressive jusqu'à la sporulation. Cette réduction, quand la relation hôte-parasite est visiblement établie, s'est révélée beaucoup plus importante que ne le laissait prévoir la surface de feuille lésée par l'infection (surface cumulée des taches infectieuses). Elle peut s'expliquer, d'une part par une altération du fonctionnement des stomates, d'autre part par une limitation de la fixation de $\mathrm{CO}_{2}$ dans les cellules du mésophylle : cette deuxième limitation étant apparemment beaucoup plus importante que la première. L'impact de $M$. brunnea sur la photosynthèse est apparu d'autant plus prononcé que la pression d'inoculum à laquelle se trouvait soumise la feuille était élevée ; il s'est avéré dépendant du génotype de l'hôte, la photosynthèse se montrant plus perturbée lorsque le clone était sensible.
\end{abstract}

Mots clés : Marssonina brunnea, Populus, photosynthèse, conductance stomatique, sensibilité.

\section{Introduction}

Marssonina brunnea (Ell. et Ev.) Magn., dont la forme parfaite est Drepanopeziza punctiformis Gremmen, est responsable de la brunissure du feuillage du peuplier, et provoque de sérieux dommages dans les plantations européennes depuis 1960. II entraîne en particulier des pertes de production estimées en Italie (vallée du Pô) à $16 \mathrm{p}$. 100 pour l'ensemble des clones et accidentellement de $60 \mathrm{p}$. 100 pour les clones les plus sensibles (Anonyme, 1980). Dès le débourrement, les feuilles se couvrent de petites taches brunes d'environ $1 \mathrm{~mm}$ de diamètre, dont le nombre augmente avec la pression d'inoculum. Ces taches s'entourent progressivement d'un halo chlorotique ; le limbe finit par jaunir et la feuille tombe prématurément. Ainsi $M$. brunnea porte atteinte au potentiel photosynthétique du peuplier, et à terme à sa production ligneuse. 
Il paraît de ce fait important d'analyser directement l'impact de l'infection sur la photosynthèse. De nombreux champignons parasites foliaires provoquent des effets dépressifs marqués sur l'assimilation nette des plantes cultivées ; c'est le cas en particulier des parasites biotrophes comme les rouilles (Mrtchell, 1979 ; Owera et al., 1981), les oïdiums (Mignucci \& Boyer, 1979 ; Gordon \& Duniway, 1982 a). Ces effets dépressifs se manifestent assez précocement après le début de l'infection : lors de l'apparition des symptômes pour les rouilles (OWERA et al., 1981) ou au moment de la sporulation des oïdiums (Gordon et Duniway, 1982 a). L'ö̈dium du chêne constitue à ce jour le seul exemple forestier abordé à propos de son effet sur la photosynthèse (HewitT et Ayres, 1975). Les réactions aux parasites nécrotrophes sont moins bien connues; on sait ainsi que la septoriose provoque des baisses de photosynthèse (Scharen \& Krupinsky, 1969). De fait il est prévisible que l'apparition des taches nécrotiques provoquées par ces parasites sur les limbes foliaires va provoquer une réduction de l'assimilation photosynthétique. Cependant nous ne savons actuellement ni à quel stade du développement de l'infection vont apparaitre ces perturbations ni quelles sont les relations quantitatives entre la réduction de la surface photosynthétiquement active et celle concomitante de l'assimilation nette de carbone.

Les réductions de photosynthèse nette observées sur feuilles infectées peuvent être au moins partiellement dues à des réductions de conductance stomatique, et donc à un ralentissement de la diffusion du $\mathrm{CO}_{2}$ gazeux vers les tissus mésophylliens ; les travaux récents sur l'oïdium (Mignucci \& Boyer, 1979 ; Gordon \& Duniway, 1982 a) montrent cependant que ces réductions de conductance n€ jouent qu'un rôle limité et que les processus mésophylliens conduisant à l'assimilation nette de $\mathrm{CO}_{2}$ sont perturbés soit par le biais d'une augmentation de respiration ou de photorespiration, soit par le biais d'une diminution de la capacité intrinsèque à fixer le $\mathrm{CO}_{2}$.

L'infection des feuilles de peuplier par $M$. irunnea n'a pour l'instant jamais été étudiée sous l'angle des conséquences physiologiques; la priorité affichée dans les programmes de recherches en France ou ailleurs portait essentiellement sur la sélection de clones résistants. L'étude des perturbations des échanges gazeux foliaires (assimilation nette de $\mathrm{CO}_{2}$ et transpiration) présentée ici a pour objectif :

- d'affiner la description des premières étapes d'installation de l'infection, en complétant les descriptions histologiques existiantes (SPIERS \& Hopcroft, 1983) ;

- de comparer ces effets primaires entre clones de sensibilité différente et ainsi d'apporter quelques éléments d'analyse des manifestations précoces de résistance au parasite.

\section{Matériel et méthodes}

\subsection{Matériel végétal}

Les plants utilisés appartiennent à trois clones de l'espèce hybride Populus $\times$ euramericana (DODE) Guinier connus pour avoir des sensibilités différentes à $M$. brunnea :

Robusta, assez résistant

I 214, sensible

Magister géant, très sensible. 
Les boutures aoûtées sont conservées l'hiver en chambre froide $\left(-7^{\circ} \mathrm{C}\right)$. Elles sont plantées sous serre au printemps en pots de 51 contenant un mélange de tourbe et de sable fertilisé. Au moment de l'inoculation, les plants ont 14 semaines, et portent 31 à 38 feuilles.

Au moins 3 jours avant les mesures de photosynthèse, les plants sont transportés en chambre climatisée $\left(16 \mathrm{~h}\right.$ par jour, $+21 \pm 1^{\circ} \mathrm{C}$ le jour et $10 \pm 1^{\circ} \mathrm{C}$ la nuit, 75 à 85 p. 100 d'humidité relative, rayonnement photosynthétiquement actif de $330 \mu$ moles de photons $\cdot \mathrm{m}^{-2} \cdot \mathrm{s}^{-1}$ au niveau des feuilles supérieures, et diminuant jusqu'à 220 $\mu$ moles pour les feuilles médianes).

\subsection{Inoculation et suivi de l'infection}

L'inoculum est constitué de suspensions de conidies prélevées sur des pustules des feuilles de "Magister géant " récoltées dans la nature, à partir du 15 mai. Celles-ci sont ajustées avec de l'eau distillée additionnée d'eau gélosée afin d'atteindre des concentrations de 50000 et 100000 conidies $\cdot \mathrm{ml}^{-1}$. Ces pressions d'inoculum ont été choisies afin d'obtenir en une inoculation un degré d'infection assez élevé comparable à celui observé en nature au milieu de la saison de végétation.

Cette suspension est pulvérisée sur la face supérieure de toutes les feuilles des plants qui sont ensuite placés à l'obscurité en atmosphère saturée en humidité pendant $24 \mathrm{~h}$.

Pour tenir compte de l'effet de l'âge des feuilles sur la sensibilité à l'infection (CEllerino et al., 1978) les mesures portent sur des feuilles d'âge équivalent auxquelles on a attribué le même numéro d'ordre suivant la procédure de DickmanN (1971) : la feuille indexée 0 est celle qui, à l'apex du plant, a au moins $2 \mathrm{~cm}$ de longueur et dont le limbe est à demi déployé. Cet index augmente d'une unité pour chaque feuille par ordre d'âge croissant : on utilise la feuille 6 pour les mesures.

La description du processus infectieux porte sur la détection des lésions puis celle de la sporulation. Le degré d'infection est estimé soit par comptage des taches, soit par mesure de la surface d'infection après décoloration par du Diméthylsulfoxyde (DMSO) à $100^{\circ} \mathrm{C}$ pendant $1 \mathrm{~h}$ (Maurer et al., 1986). Cette procédure permet d'englober dans l'estimation de la surface infectée la nécrose elle-même ainsi que le halo chlorotique qui l'entoure.

Deux boutures sont utilisées par traitement pour suivre l'évolution de l'infection : les mesures de photosynthèse sont réalisées sur la $6^{\mathrm{e}}$ feuille de l'une d'entre elles.

\subsection{Mesures des échanges gazeux}

Le suivi de photosynthèse débute à partir du jour précédant l'inoculation, et se poursuit par une mesure quotidienne sur la même feuille pendant 15 jours.

Ces mesures sont réalisées simultanément dans trois chambres d'assimilation en "altuglass" ( 61 de volume intérieur) montées en parallèle sur un même système de régulation. L'ensemble constitue un circuit ouvert régulé en température dans les chambres, et en humidité et $\mathrm{CO}_{2}$ à l'entrée des chambres. 
La température de l'air dans les chambres est mesurée par des thermomètres à mercure, et régulée par des éléments Peltier $\left(22 \pm 0,5^{\circ} \mathrm{C}\right)$. Un ventilateur radial permet un brassage constant de l'air ; le débit général dans le circuit est maintenu à $150 \mathrm{I} \cdot \mathrm{h}^{-1}$. Le rayonnement incident est assuré par des lampes à vapeur de sodium (Sont $400 \mathrm{~W}$ Philips) équipées d'un réflecteur parabolique; il est ajusté à 390 moles $\cdot \mathrm{m}^{-2} \cdot \mathrm{s}^{1} \quad( \pm 5)$ dans le rayonnement photosynthétique actif (PAR : $400-700 \mathrm{~nm})$.

La concentration en $\mathrm{CO}_{2}$ à l'entrée des chambres est mesurée à l'aide d'un analyseur infrarouge $\mathrm{ADC} \mathrm{MK}$ II et maintenue à $350 \pm 2 \mu$ moles $\mathrm{CO}_{2} \cdot$ mole $^{-1}$; les concentrations réelles dans les chambres varient de ce fait suivant la capacité d'assimilation des feuilles entre 250 et $320 \mu$ moles - mole '. L'humidité de l'air est mesurée à l'aide d'un hygromètre à point de rosée General Eastern $1100 \mathrm{DP}\left( \pm 0,05{ }^{\circ} \mathrm{C}\right)$ et est régulée à l'entrée de manière à obtenir dans les chambres, compte tenu de l'apport transpiratoire, $65 \pm 10$ p. 100 d'humidité relative.

La différence de concentration en $\mathrm{CO}_{2}$ entre l'entrée et la sortie de la chambre, permettant le calcul de l'assimilation nette, est mesurée par un analyseur ADC MK III en mode différentiel, et la transpiration par mesures alternées de l'humidité absolue à l'entrée et la sortie.

La feuille est photographiée quotidiennement afin d'estimer sa surface à l'aide d'un analyseur d'images Leitz. Un système d'électrovannes permet de mesurer les paramètres relatifs aux échanges gazeux successivement dans les trois chambres. Les mesures concernent des situations de régime permanent et. nécessitent donc une phase d'équilibrage d'environ 90 minutes.

\subsection{Calculs}

Les mesures d'échanges gazeux permettent le calcul direct de $A$ (assimilation nette de $\mathrm{CO}_{2}, \mu$ moles $\mathrm{CO}_{2} \cdot \mathrm{m}^{-2} \cdot \mathrm{s}^{-1}$ ) et de $\mathrm{E}$ (transpiration, en mmoles $\mathrm{H}_{2} \mathrm{O} \cdot \mathrm{m}^{-2} \cdot \mathrm{s}^{-1}$ ). A partir de ces données. on applique un modèle de diffusion de $\mathrm{CO}_{2}$ et de vapeur d'eau dérivé de celui de GaASTra (1959) et fréquemment repris depuis (JARvis, 1971).

- Calcul de la conductance foliaire à la vapeur d'eau

$$
\mathrm{g}^{\mathrm{H}_{2} \mathrm{O}}=\frac{E}{\mathrm{C}_{\mathrm{i}}^{\mathrm{H}_{2} \mathrm{O}}-\mathrm{C}_{\mathrm{a}}^{\mathrm{H}_{2} \mathrm{O}}}
$$

$\mathrm{C}_{\mathrm{i}}^{\mathrm{H}_{2} \mathrm{O}}$ : concentration en vapeur d'eau dans la cavité sous stomatique (mmoles/ mole ${ }^{-1}$ ) estimée comme étant la concentration de vapeur d'eau saturante à la température de la feuille (égale à celle de l'air dans la chambre) ;

$\mathrm{C}_{\mathrm{a}} \mathrm{H}_{2} \mathrm{O}$ : concentration en vapeur d'eau dans l'air ;

$\mathrm{g}^{\mathrm{H} z \mathrm{O}}$ : conductance foliaire équivalente pour la vapeur d'eau (mmoles $\cdot \mathrm{m}^{-2} \cdot \mathrm{s}^{-1}$, Cowan, 1977)

avec $g^{\mathrm{H}_{2} \mathrm{O}}=\frac{1}{r_{\mathrm{a}}+\mathrm{r}_{\mathrm{s}}}+\frac{1}{\mathrm{r}_{\mathrm{c}}} \simeq \frac{1}{\mathrm{r}_{\mathrm{a}}+\mathrm{r}_{\mathrm{s}}} ;$

$r_{a}$ : résistance aérodynamique mesurée à l'aide d'un buvard découpé à la forme d'une feuille de peuplier et évaporant librement dans la chambre ; $r_{a} \simeq 2,8$ $10^{-2} \mathrm{~m}^{2} \mathrm{~s} \cdot$ mmole $^{-1}$, dans les conditions de l'expérience ; 
$r_{\mathrm{s}} \quad$ : résistance stomatique proprement dite ;

$r_{c} \quad$ : résistance cuticulaire infinie.

- Calcul de la conductance équivalente pour le $\mathrm{CO}_{2}$

$$
g=\frac{g^{112()}}{1,6}\left(\text { JARVIS, }_{1971)}\right.
$$

Dans les conditions de nos expériences (22 " $\mathrm{C}$ et pression atmosphérique) on peut convertir ces unités de conductance en unités classiques avec la relation :

$1 \mathrm{mmole} \cdot \mathrm{m}^{2} \cdot \mathrm{s}^{1}=0,025 \cdot 10^{-3} \mathrm{~ms}^{-1}$

- Calcul de la concentration en $\mathrm{CO}_{2}$ des espaces intercellulaires

$$
\mathrm{C}_{\mathrm{i}}^{\mathrm{CO}_{2}}=\mathrm{C}_{\mathrm{i}}^{\mathrm{CO}} \mathrm{O}_{2}-\frac{\mathrm{A}}{\mathrm{g}}(\text { CowaN, 1977) }
$$

$\mathrm{C}_{\mathrm{a}}^{\mathrm{CO}_{2}}$ et $\mathrm{C}_{\mathrm{i}}^{\mathrm{CO}_{2}}$ : concentrations molaires en $\mathrm{CO}_{2}$ respectivement dans l'air et dans les espaces intercellulaires, la première étant mesurée directement par un analyseur à infrarouge.

Les feuilles de peuplier sont amphistomatiques (LARSEN, 1968), gs constitue donc une conductance équivalente calculée à l'échelle de la feuille entière.

Ce modèle de diffusion est appliqué sans modification à des feuilles infectées par M. brunnea. En effet:

- il n'y a pas, contrairement au cas des oïdiums (Gordon \& Duniway, 1982 a) de développement de mycélium en surface des feuilles (SpIERs \& Hopcroft, 1983) : la résistance aérodynamique à la diffusion n'est donc pas modifiée, et aucun flux transpiratoire directement originaire du mycélium ne perturbe la mesure,

- les acervules se développent sous l'épiderme (SpiErs \& Hopcroft, 1983) dont la rupture n'intervient que très tardivement; jusqu'à cette rupture, les variations de conductance foliaire reflètent strictement celles de l'ouverture stomatique.

Du fait du système de mesure utilisé, les valeurs d'assimilation nette de $\mathrm{CO}$, ont été obtenues avec des concentrations en $\mathrm{CO}_{2}$ de l'air variant entre 280 et 340 $\mu$ moles $\cdot$ moles $^{-1}$. Une transformation est donc nécessaire pour les rendre strictement comparables. Cette transformation est effectuée en utilisant une représentation de $A$ en fonction de $\mathrm{C}_{\mathrm{i}}$ (Jones, 1973) (fig. 2) sur laquelle chaque valeur de $\mathrm{A}$ apparait comme l'intersection d'une «fonction de demande" $A=f\left(C_{i}\right)$ passant par le point de compensation en $\mathrm{CO}_{2}(\mathrm{r})$ et d'une droite d'équation $\mathrm{A}=-\mathrm{g}\left(\mathrm{C}_{\mathrm{i}} \mathrm{CO}_{2}-\mathrm{C}_{\mathrm{a}}{ }^{\mathrm{CO}_{2}}\right)$ appelée «fonction de fourniture" (Jones, 1973 ; Farquhar \& Sharkey, 1982).

Deux types de transformation sont effectués à partir de cette représentation :

- toutes les mesures sont rapportées à un $\mathrm{C}_{\mathrm{a}}$ unique de $300 \mu$ moles $\cdot$ moles $^{-1}$ par déplacement sur les fonctions de demande tout en maintenant une conductance constante (fig. 2a);

- toutes les mesures sont rapportées à un $\mathrm{C}_{i}$ unique de $200 \mu$ moles $\cdot$ mole $^{-1}$ sans se préoccuper de la conductance stomatique (fig. 2b). Cette deuxième transformation permet de comparer les activités photosynthétiques foliaires en s'affranchissant des limitations imposées à la diffusion du $\mathrm{CO}_{2}$ dans les feuilles par les stomates (fig. $2 \mathrm{~b}$ ) . 


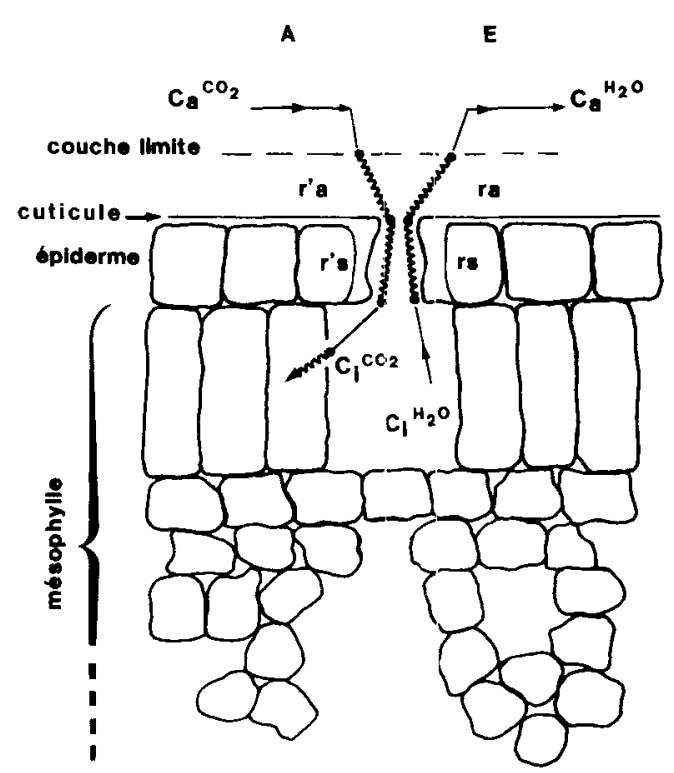

FIG. 1

Echanges gazeux foliaires : localisation et signification des résistances à la diffusion.

Foliar gas exchange: localisation and significance of resistances to $\mathrm{CO}_{2}$ diffusion.

Symboles A Symbols :

$\mathrm{E}$<smiles>[CH][CH][CH2]</smiles>
$C^{1120} C_{1100}$ :

$r_{i,}^{\prime}, r_{i s}$

$r^{\prime}, r$ $g=\frac{1}{r^{\prime}{ }_{1}+r^{\prime}}$
: assimilation nette de $\mathrm{CO},\left(\mu\right.$ moles $\left.\cdot \mathrm{m}^{2} \cdot \mathrm{s}^{-1}\right) /$ net $\mathrm{CO}$, assimilation rate (umoles. $m^{2} \cdot s$ ')

: transpiration [flux net de vapeur d'eau sortant par les stomates (mmoles $\left.\cdot \mathrm{m}^{2} \cdot \mathrm{s}^{\prime}\right) \mid /$ transpiration /net water vapour flow through stomata (mmoles $\left.\cdot m^{2} \cdot s^{\prime}\right)$ )

concentration en $\mathrm{CO}$, e? vapeur d'eau, respectivement dans l'atmosphère, et dans les espaces intercellulaires du mésophylle ( $\mu$ moles. mole ') / $\mathrm{CO}$, and water vapour concentrations, respectively in the atmosphere and in the mesophyll intercellular spaces ( $\mu$ moles $\cdot$ mole ${ }^{-i}$ )

: résistance aérodynamiqus: à la diffusion de $\mathrm{CO}$, et de vapeur d'eau respectivement $\left(\mathrm{m}^{2} \mathrm{~s} \cdot\right.$ mmole 1$) /$ aerodynamic resistances respectively to $\mathrm{CO}_{2}$ and water vapour diffusion $\left(\mathrm{m}^{2} \mathrm{~s} \cdot \mathrm{mmole}^{-1}\right)$

: résistance stomatique a la diffusion de $\mathrm{CO}_{2}$ et de vapeur d'eau respectivement $\left(\mathrm{m}^{2} \mathrm{~s} \cdot\right.$ mmole ') / siomatal resistances respectively to $\mathrm{CO}_{2}$ and water vapour diffusion $\left(\mathrm{m}^{2} s \cdot\right.$ mmole $\left.^{-1}\right)$

: conductance equivalente de la feuille au $\mathrm{CO}_{2}\left(\right.$ mmole $\left.\cdot \mathrm{m}^{2} \cdot \mathrm{s}^{-1}\right) /$ equivalent leaf conductance to $\mathrm{CO}_{2}\left(\mathrm{mmole} \cdot \mathrm{m}^{2} \cdot \mathrm{s}^{-1}\right)$

Par hypothèse, $C_{i}^{1 \mid 2(0)}$ est assimilé à l'humidité saturante à la température de la feuille $; r^{\prime}{ }_{A}$ et $r_{A}$ restent constantes pour unc forme de feuille donnée dans nos chambres de mesures / $B y$ hypothesis, $C_{i}^{\prime l(O)}$ is assimilated to saturated humidity at leaf temperature; $r^{\prime}$ et $r$ are constant for a given leaf shape in our gas exchange chamber. 

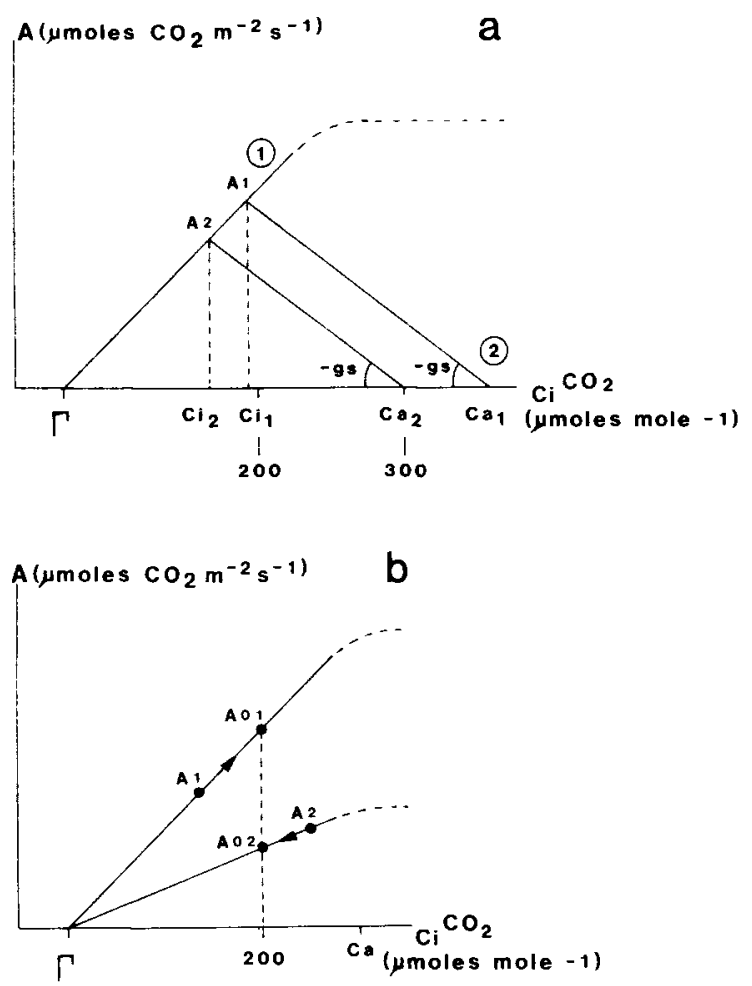

FIG. 2

Analyse graphique des résultats d'assimilation nette (A) en fonction de la concentration interne en $\mathrm{CO}_{2}\left(\mathrm{C}_{i}{ }^{\left(\mathrm{O}_{2}\right)}\right.$ et de la conductance stomatique $\left(g_{s}\right)$.

Graphic analysis of net $\mathrm{CO}_{2}$ assimilation rates (A) as a function of internal $\mathrm{CO}_{2}$ concentration $\left(\mathrm{C}_{i}^{\left(\mathrm{O}_{2}\right.}\right)$ and of stomatal conductance.

1 = fonction de demande, linéaire jusqu'à 200 umoles $\mathrm{CO}_{2} \cdot$ mole $1 /$ demand function. linear till $C_{i}=200$ umoles . mole

$2=$ fonction de fourniture, de pente $-\mathrm{g} /$ supply function, slope $=-g$.

Une première transformation permet de passer de $\mathrm{Ca}_{1}$ à un $\mathrm{Ca}$ de référence $(300$ $\mu$ moles $\cdot$ molc $\left.^{-1}\right)$ et à l'assimilation nette correspondante $\left(\mathrm{A}_{1}-\mathrm{A}_{2}\right) / A$ first transformation was performed between $\mathrm{Ca}_{1}$ and $\mathrm{Ca}$, at $300 \mu$ moles $\cdot$ mole $^{-1}$ and consequently between $\mathrm{A}_{1}$ et $A_{2}$.

$\Gamma=$ point de compensation en $\mathrm{CO}_{2} / \mathrm{CO}_{2}$ compensation point.

Unc seconde transposition permet de rapporter tous les résultats à une valeur de référence $\mathrm{C}_{\mathrm{i}}$ de 200 umoles - mole ' et de s'affranchir ainsi des conductances stomatiques / A second transformation was performed in order to relate all the results to $C_{i}$ of 200 umoles -mole', and so to leave the effects of stomatal conductance out of account. 
Des essais préliminaires (Maurer, 1985) ont permis de montrer que les courbes de réponse au $\mathrm{C}_{\mathrm{i}}$ (fonction de fourniture) sont quasi linéaires jusqu'aux concentrations utilisées dans notre travail. De même, on a pu constater que le point de compensation était relativement constant et voisin de $35 \mu$ moles $\cdot$ mole $^{-1}$. Ces deux hypothèses (linéarité de la fonction de demande et constance de $\Gamma$ ) sont étendues aux feuilles infectées ; les limites de validité de cette démarche seront discutées après présentation des résultats.

\section{Résultats}

\subsection{Développement de linfaction (tabl. 1)}

Sur la $6^{\text {e }}$ feuille des taches infectieuses sont détectées 6 ou 7 jours après l'inoculation. Elles se transforment en nécroses entourées d'un halo chlorotique trois à quatre jours plus tard. Au quinzième jour les acervules sont parfaitement constitués mais dans aucun cas n'est survenue la rupture de l'épiderme. En ce qui concerne "Robusta », les feuilles se nécrosent progressivement sous la plus forte pression d'inoculum.

TABI.EAU 1

Comparaison des principales caractéristiques des réponses foliaires à l'infection par M. brunnea sur les trois clones de peuplier.

Comparison of the main characteristics of foliar reactions to infection by M. brunnea on three poplar clones. Data shown, are:

- time lag (days) between inoculation and appearance of necrotic spots

- relative area of necrotic spots ( $p .100$ of total leaf area)

- relative sporulation rate (in p. 100 of toial necrotic spots).

\begin{tabular}{|c|c|c|c|c|c|c|}
\hline Clone & \multicolumn{2}{|c|}{ «Robusta» } & \multicolumn{2}{|c|}{ «I-214 » } & \multicolumn{2}{|c|}{ «Magister gćant» } \\
\hline $\begin{array}{c}\text { Pression d'inoculum conidies } \\
\mathrm{ml}^{+} \ldots \ldots \ldots \ldots \ldots \ldots\end{array}$ & 50000 & 100000 & .50000 & 100000 & 50000 & 100000 \\
\hline $\begin{array}{l}\text { Délai d'apparition des taches } \\
\text { d'infection après inoculation } \\
\text { (jours) . . . . . . . }\end{array}$ & 6 & 6 & 7 & 6 & 7 & 6 \\
\hline $\begin{array}{c}\text { Surface des taches }(\% \text { de la } \\
\text { surface foliaire }) \ldots \ldots \ldots\end{array}$ & 16 & 60 & 11 & 22 & 14 & 27 \\
\hline $\begin{array}{l}\text { Taux de sporulation par rap- } \\
\text { port au nombre de taches } \\
\text { (jour 16) } \ldots \ldots \ldots \ldots \ldots\end{array}$ & 0 & $\begin{array}{l}100 \\
\text { nécrose } \\
\text { rapide }\end{array}$ & $20-30$ & $\begin{array}{l}100 \\
\text { jaunis- } \\
\text { sement } \\
\text { rapide }\end{array}$ & 100 & 100 \\
\hline
\end{tabular}

\subsection{Evolution de l'assimilation nette et a'e la conductance stomatique}

\subsection{Témoin (fig. $3 a$ et b)}

Les trois clones présentent des niveaux d'assimilation nette (rapportée à $\mathrm{C}_{i:}^{\left(\omega_{2}\right.}=300 \mu$ moles $\cdot$ mole $\left.{ }^{-1}\right)$ et de conductance stomatique assez différents; le clone le 
plus efficient est « I-214» (A et $\mathrm{g}$ variant entre 10 et $15 \mu$ moles $\mathrm{CO}_{2} \cdot \mathrm{m}^{-2} \cdot \mathrm{s}$ ' et 150 à $200 \mathrm{mmoles} \cdot \mathrm{m}^{-2} \cdot \mathrm{s}{ }^{1}$ respectivement) suivi de «Robusta » et de "Magister géant » (A et $\mathrm{g}$ proches de $10 \mu$ moles $\mathrm{CO}_{2} \cdot \mathrm{m}^{-2} \cdot \mathrm{s}^{-1}$ et 100 mmoles $\cdot \mathrm{m}^{-2} \cdot \mathrm{s}^{-1}$ respectivement). Le classement est identique en ce qui concerne la conductance stomatique.

On constate par ailleurs que A et $\mathrm{g}$ augmentent avec l'âge de la feuille de façon très marquée, en particulier sur « I-214».

\subsection{Feuilles infectées (fig. $3 a$ et b)}

L'évolution de l'assimilation nette et de la conductance stomatique apparaît fortement perturbée chez les trois clones sous les deux pressions d'inoculum. Les perturbations se traduisent soit par une chute progressive $\left(50000\right.$ conidies $\left.\cdot \mathrm{ml}^{-1}\right)$ ou très rapide (100000 conidies $\cdot \mathrm{ml}^{-1}$ ) des niveaux d'assimilation; on ne retrouve dans aucun cas l'augmentation observée sur les témoins. Ces phénomènes se produisent très précocement (3 à 4 jours après l'inoculation), bien avant l'expression des symptômes. Dans certains cas ( «I-214 » surtout) apparaît une période transitoire pendant laquelle la diminution de $\mathrm{A}$ et $\mathrm{g}$ est très forte suivie d'un léger rétablissement puis d'une diminution beaucoup plus progressive.

En rapportant l'assimilation nette à une valeur constante $\left(200 \mu\right.$ moles $\cdot$ mole $\left.^{-1}\right)$ de $\mathrm{C}_{i}$, et en l'exprimant en pourcentage de celle des témoins, il devient possible d'apprécier l'importance des limitations d'origine mésophylienne dans la réduction de l'assimilation nette (fig. 4). On constate ainsi plusieurs faits marquants :

- à cette valeur de $C_{i}$, l'assimilation nette des feuilles infectées est toujours inférieure à celle des témoins, ce qui traduit clairement une moindre capacité des tissus mésophylliens à fixer le $\mathrm{CO}_{2}$ en cas d'infection. La réduction de conductance stomatique ne peut en aucun cas être tenue pour responsable de la réduction de l'assimilation nette ;

- cette perturbation du fonctionnement mésophyllien est précoce puisqu'elle apparaît avant l'extériorisation des symptômes après deux à trois jours d'incubation ;

- la réduction est plus importante avec la pression d'inoculum la plus forte ;

- le pic d'assimilation nette relative que présente «Robusta» (fig. 4a) est un artefact résultant d'un mauvais fonctionnement passager de la feuille témoin.

De plus, on constate l'existence de deux phases dans la cinétique de l'assimilation nette après inoculation (fig. 4) ;

- dans un premier temps après 4 jours, et ceci très nettement dans cinq cas sur six, se produit une réduction brutale de la capacité à fixer le $\mathrm{CO}_{2}$ par rapport au témoin. Cette phase se termine sensiblement lors de l'apparition des taches, avec quelques nuances entre les clones;

- dans une seconde étape, se manifeste une stabilité relative ou tout du moins une réduction peu marquée. De plus, la sporulation constatée chez "Magister géant " et «I-214 »n'a pas provoqué de modification de la capacité photosynthétique.

\subsection{Liaison entre surface infectée et réduction de l'assimilation nette}

La baisse de photosynthèse ne peut pas être attribuée uniquement à la réduction de la surface active des feuilles (nécroses et dépigmentation) : par exemple à $20 \mathrm{p} .100$ environ de surface infectée correspond en fait une chute de plus de 50 p. 100 de la photosynthèse ("Robusta " et "Magister géant» à 50000 spores $/ \mathrm{ml}$ ). Une telle distorsion est apparue dans tous les cas (fig. 5). 

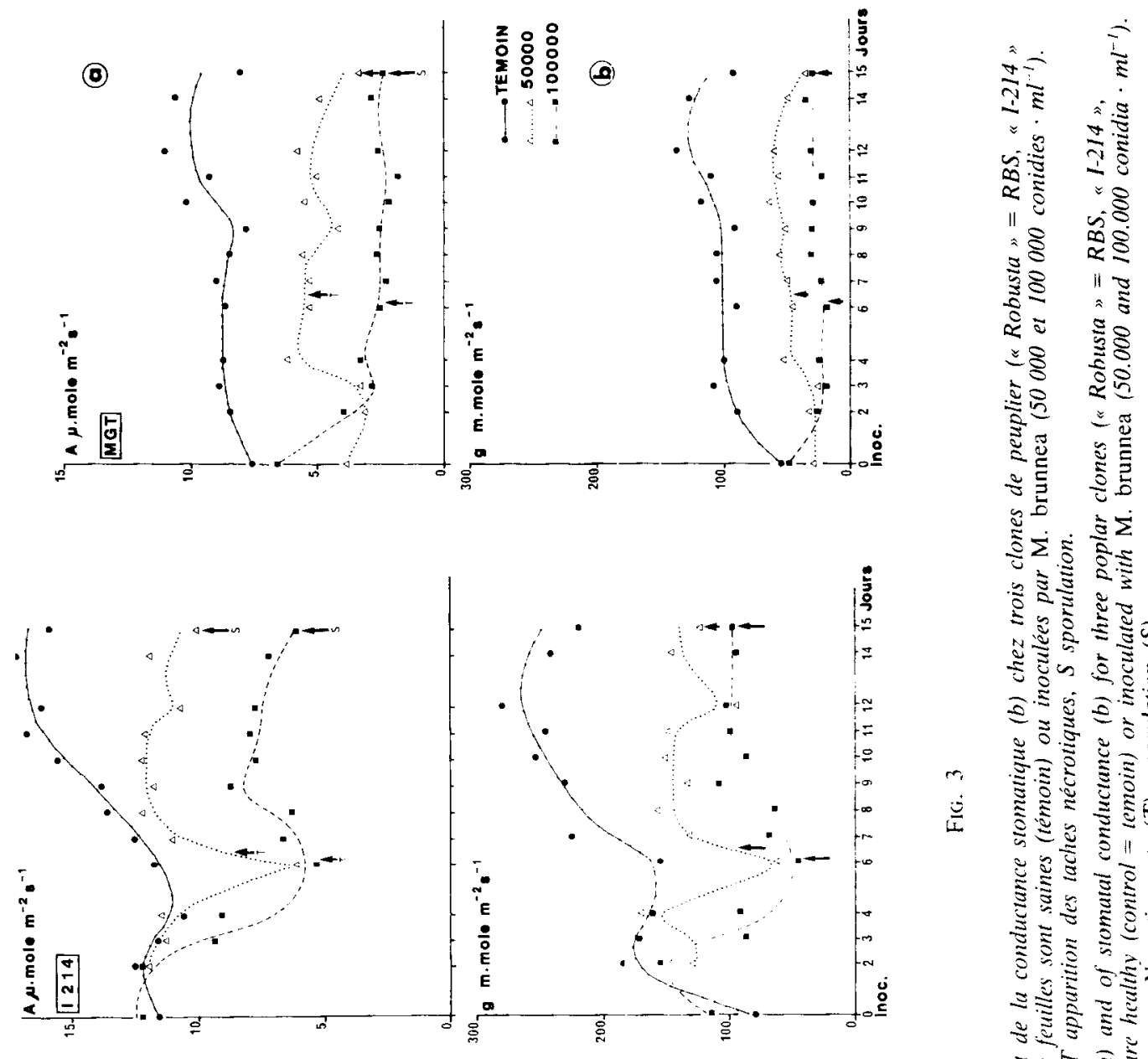

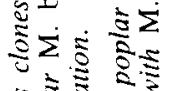

3ฐ

$\checkmark)$

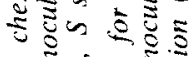

อ

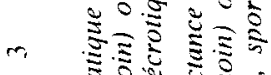

立

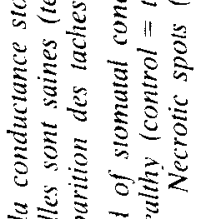

$\Xi \underset{\Im}{\Xi}$

यू⿵

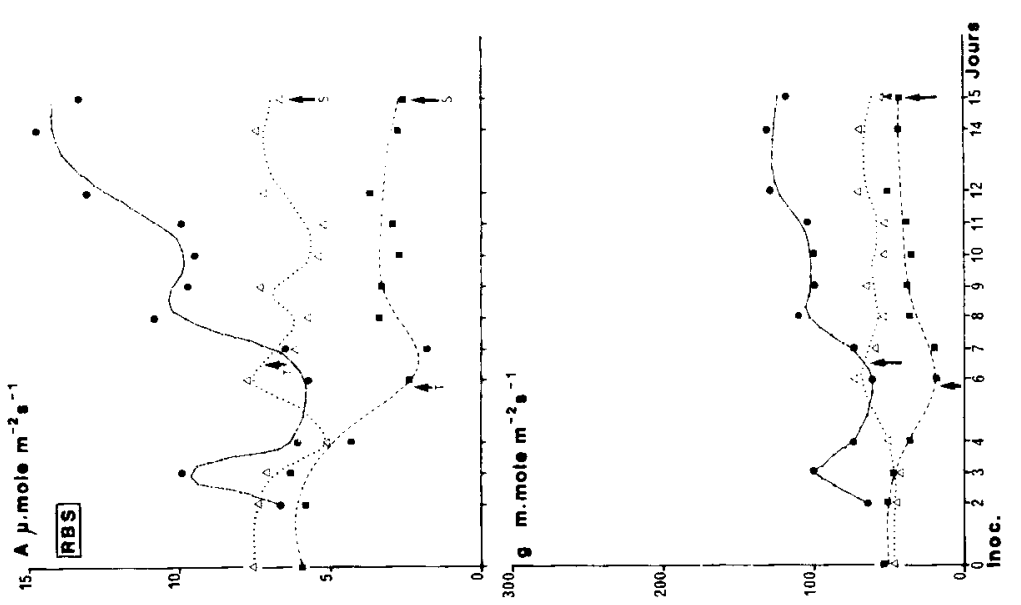

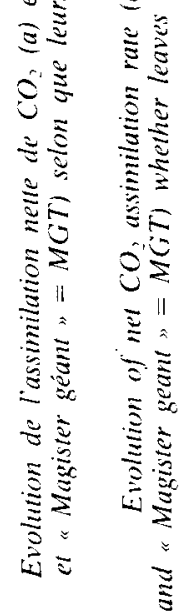




\subsection{Comparaison entre clones}

L'analyse des résultats présentée jusqu'ici permet de mettre en relief des différences importantes de comportement entre clones. Elles apparaissent à plusieurs niveaux :

- degré d'infection et sporulation. «Robusta» présente un comportement très distinct de celui des autres clones : sous la pression d'inoculum la plus faible aucune sporulation n'intervient. Par contre, sous la pression la plus forte, il apparaît le plus infecté. La sporulation est généralisée mais suivie rapidement de la nécrose totale de la feuille. En terme d'infection "I-214 » et «Magister géant " ne se distinguent pas nettement. A faible pression d'inoculum, la sporulation n'est que partielle chez «I$214 »($ tabl. 1) ;
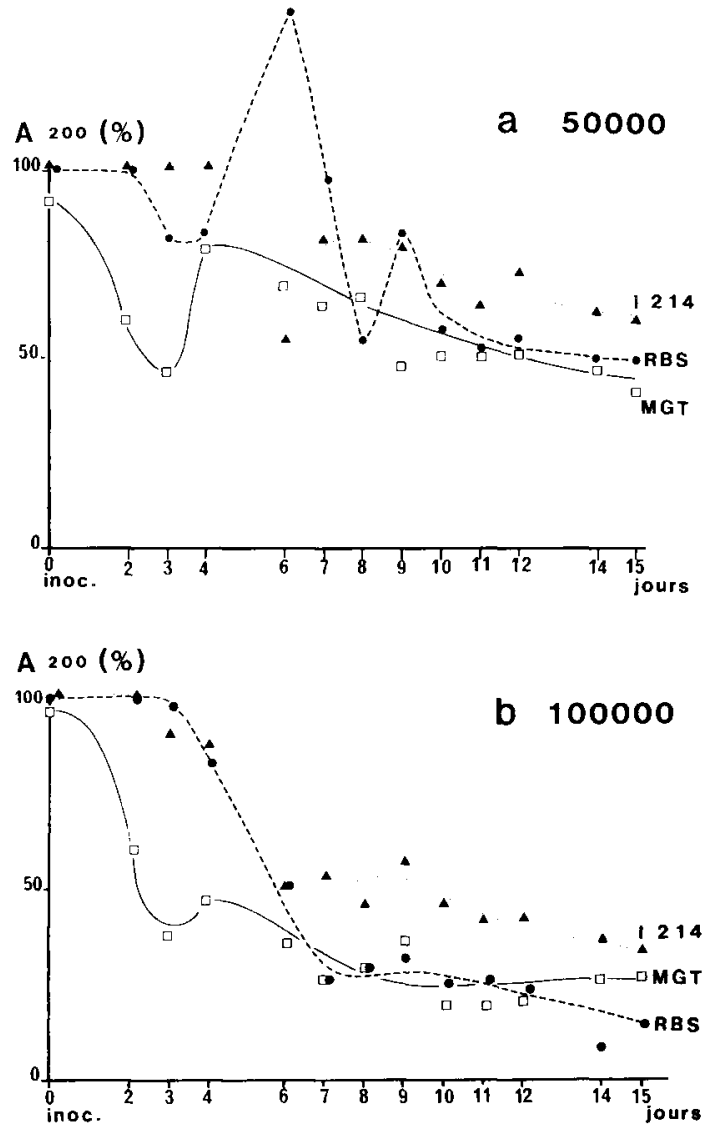

FIG, 4

Evolution de l'assimilation nette rapportée à $C_{i}=200 \mu$ moles $\cdot$ mole ${ }^{-1}$ des feuilles inoculées et exprimée en pourcentage des témoins sains: $a=50000$ conidies $\cdot m l^{-1}, b=100000$ conidies $\cdot \mathrm{ml}^{-1}$

Evolution of net assimilation rate at $C_{i}=200 \mu$ moles $\cdot$ mole ${ }^{-1}$ for inoculated leaves. Results are estimated as percent of healthy control rates: $a: 50.000$ conidia $\cdot \mathrm{ml}^{-1}, \mathrm{~b}: 100.000$ conidia $\cdot \mathrm{ml}^{-1}$. 
- la précocité des perturbations de la photosynthèse (fig. 4) : les réactions les plus rapides se produisent sur le clone "Magister géant" avec une baisse d'activité dès le $2^{\text {e }}$ jour d'inoculation, la latence est légèrement plus grande sur « Robusta » et «I-214 »;

- l'importance de la réduction d'assimilation nette (fig. 4) : sous les deux pressions d'inoculum, les réductions sont plus marquées sur les deux clones "Magister géant » et « Robusta » que sur «I-214»;

- l'importance relative des nécroses et de la réduction d'assimilation nette (fig. 5 et tabl. 2) : le rapport

réduction d'assimilation nette

surface nécrosée

permet de distinguer les trois clones. Sous faible pression d'inoculum, "Robusta " et «I-214 s subissent une perte comparable mais irférieure à celle affectant « Magister géant». Lorsque la pression d'inoculum est plus forte, «Robusta » présente une moindre perturbation relative que les deux autres clones.

Tableat 2

Rapport entre les réductions d'assimilation n'tle $\left(C_{i}=200 \mu\right.$ moles $\cdot$ mole $\left.{ }^{-1}\right)$ et la surface infectée pour les clones aux deux pressions dinoculum.

Ratios of reduction of net assimilation rates at $C_{i}=200 \mu$ moles $\cdot$ mole ${ }^{-1}$ to infected leaf area for the three clones under the two inoculum pressures.

\begin{tabular}{c|c|c|c}
\hline Inoculum & "Robusta » & «I-214» & « Magister géant » \\
\hline 50000 & 3.0 & 3,0 & 3,8 \\
\hline 100000 & 1,7 & 2,5 & 2,8 \\
\hline
\end{tabular}

\section{Discussion}

L'évolution de l'assimilation nette de $\mathrm{CO}_{2}$ et de la conductance stomatique observée sur les feuilles de peuplier infectées par $M$. brunnea soulève trois questions essentielles :

1) parmi les processus impliqués dans l'assimilation nette de $\mathrm{CO}_{2}$ (diffusion gazeuse du $\mathrm{CO}_{2}$, carboxylation, respirations...) lesquels sont plus particulièrement perturbés par l'infection?

2) les différentes phases de la perturbation du fonctionnement photosynthétique correspondent-elles à des étapes de la relation hôte-parasite ?

3) comment peut-on expliquer l'absence de relation directe entre la réduction d'assimilation nette et celle de la surface foliaire active?

Une diminution d'origine infectieuse de l'assimilation nette de $\mathrm{CO}_{2}$ peut a priori résulter de phénomènes multiples isolés ou synergiques: diminution de la conductance stomatique, augmentation de la respiration, diminution de l'activité carboxylase ou 


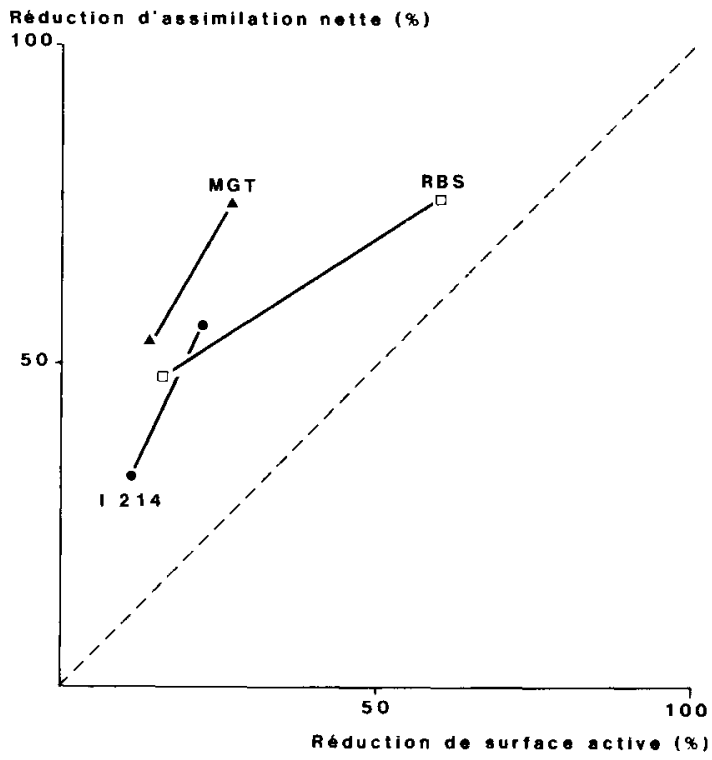

FIG. 5

Relation entre la réduction de la surface assimilatrice des feuilles et celle de l'assimilation nette (pour $C_{1}=200 \mu$ moles $\cdot$ mole ${ }^{-1}$ ) des feuilles infectées des trois clones ( $1-214 "$, "Robusta " = RBS, "Magister géant " = MGT).

Relation between reduction of foliar assimilating area and decrease in net assimilation rate at $C_{i}=200 \mu$ moles $\cdot$ mole ${ }^{-1}$ on infected leaves of three clones ( I-214 », "Robusta » = RBS, " Magister geant »= MGT).

augmentation de l'activité oxygénase de la Ribulose biphosphate carboxylase oxygénase (Rubisco) des cellules mésophylliennes. Dans le cas de l'infection par $M$. brunnea il est indéniable que la conductance stomatique est altérée chez l'hôte de la même façon que cela a déjà été décrit à propos de nombreux parasites foliaires dont Erysiphe polygoni (Gordon \& Duniway, 1982 b) et Uromyces phaseoli (Duniway \& Durbin, 1971) : la réduction de conductance stomatique correspondrait surtout à un blocage des mouvements ostiolaires, ce qui peut augmenter fortement la sensibilité à des contraintes hydriques (Gordon \& Duniway, 1982 b). Ce blocage est parfois le fait d'une toxine fongique, telle l'ochracine (Bousquet et al., 1978). Les calculs que nous avons effectués à concentration interne de $\mathrm{CO}_{2}$ fixée ont montré que cet effet stomatique ne pouvait à lui seul rendre compte de l'ensemble de la diminution de l'assimilation nette. Une partie notable des perturbations de l'assimilation nette constatées ici est donc d'origine mésophyllienne, au sens large.

L'analyse des mécanismes provoquant la réduction d'assimilation nette peut être abordée en utilisant les termes du bilan global d'assimilation d'une feuille : somme des pertes de $\mathrm{CO}_{2}$ par la respiration obscure (Rd), la photorespiration (RI) et des gains de $\mathrm{CO}_{2}$ du fait de la photosynthèse réelle $(\mathrm{Ag})$.

Deux éléments permettent de préciser les termes de ce bilan : 
- Ie bilan respiratoire n'est certainement pas modifié de manière significative par la respiration propre du champignon compte tenu du rapport des biomasses foliaires et mycéliennes en présence ;

- par ailleurs, quelques mesures partielles (MAURER, 1985) mettent en évidence une relative stabilité du point de compensation en $\mathrm{CO}_{2}(\Gamma)$, c'est-à-dire de la production respiratoire de $\mathrm{CO}_{2}$ par la feuille infectée, au moins dans les premiers jours de l'infection (jusqu'à l'apparition des symptômes).

Nous sommes ainsi amenés à penser que les réductions observées proviennent en grande partie d'une dégradation de la capacité des tissus mésophylliens à fixer le $\mathrm{CO}_{2}$ (Ag).

Les indications contradictoires apportées par la littérature ne permettent pas de préciser cette conclusion. En effet, tous les cas de figure possibles apparaissent, et ce sans liaison avec le caractère nécrotrophe ou biotrophe du parasite: auglinentation concomittante de Rl et Rd avec Erysiphe pisi (Ayres, 1976), Puccinia hordei (Owera et al., 1981), ou Erysiphe graminis spp. hordei (WA.LTERS \& AYRES, 1983), augmentation de $\mathrm{Rd}$ accompagnée soit d'une diminution de RI (Erysiphe polygoni, Gordon \& Duniway, 1982 a) soit d'une relative stabilité de RI (Puccinia graminis, Mitchell, 1979 ) et enfin stabilité des deux formes de respiration (Microsphaera diffusa, Mignucci \& BOYER, 1979).

Même si nous ne pouvons en l'état actuel la préciser d'un point de vue quantitatif, la capacité propre du mésophylle à fixer le $\mathrm{CO}_{2}(\mathrm{Ag})$ est réduite par une infection ; de nombreux mécanismes ont dailleurs été rendus responsables de cette réduction: altération structurale de chloroplastes, destruction de chlorophylle, dégradation de la Rubisco.

Signalons enfin que l'infection par des parasites provoquant des symptômes localisés (rouilles, $M$. brunnea) introduit un biais dans ce raisonnement. En effet, si la feuille saine peut être considérée comme présentant une relative homogénéité de fonctionnement sur toute sa surface, il n'en va plus de même pour une feuille à taches nécrotiques : les termes du bilan du carbone ne sont plus les mêmes dans les taches et les zones apparemment saines.

Quel que soit le clone, et la pression d'incoculum qu'il subit, les perturbations de la photosynthèse présentent une cinétique comparable comportant plusieurs phases. Deux à trois jours après l'inoculation nous avons détecté une diminution simultanée de l'assimilation nette et de la conductance stomatique. A ce stade aucun symptôme n'est visible. Un phénomène semblable a été décrit à propos de l'assimilation nette sous l'effet d'Erysiphe polygoni (GoRdon \& Duniw'AY, 1982 a) et d'Helminthosporium teres (Rowe \& REID, 1979). Après l'infection par Puccinia hordei, la conductance stomatique est affectée précocement ainsi que nous l'avons constaté ici. Nos résultats peuvent être rapprochés de la description histologique proposée par SpIERs \& Hopcroft (1983) même si ces auteurs ont choisi des conditions expérimentales différentes (disques foliaires en survie). Ils signalent ainsi que dans les premiers jours, seules les cellules épidermiques sont colonisées par $M$. brunnea. Cet envahissement superficiel, qui présente des analogies avec celui des oïdiums, suffit donc pour initier des perturbations notables de la photosynthèse dans le mésophylle. Celles-ci s'accélèrent les jours suivants et l'assimilation nette de $\mathrm{CO}_{2}$ passe par un minimum qu'on peut situer au moment de l'envahissement du mésophylle tel que décrit par Spiers \& Hopcroft (1983). Cette phase initiale de réduction est suivie par une stabilité relative voire même dans certains cas par un rétablissement sensible de la capacité photosynthétique. La transition se situe un peu 
avant (« Magister géant »), pendant ( «I-214») ou légèrement après (《Robusta ») l'apparition des nécroses. A l'inverse des autres Marssonina du peuplier, les nécroses de $M$. brunnea ne s'étendent guère après leur apparition. Tout se passe comme si l'hôte, même jugé sensible, s'opposait à l'expansion du mycélium, ce qui peut être interprété comme une réaction, certes tardive, de défense. Nous savons à présent que cette restriction du parasite est concomitante du rétablissement partiel de la photosynthèse, ou au moins d'un arrêt de sa dégradation.

Il est remarquable de constater que pour tous les clones, le dommage subi par la photosynthèse nette excède largement celui exprimé en pourcentage de surface foliaire lésée (nécroses et halos chlorotiques). Il se pourrait certes que notre méthode de mesure (Maurer et al., 1986) sous-estime l'importance des lésions. Cependant le cas du Magister géant ( 27 p. 100 de surface lésée et 75 p. 100 de réduction de la photosynthèse nette) nous fait penser que l'erreur sur l'estimation de l'infection ne joue pas un grand rôle dans l'explication de la distorsion. Les auteurs ayant mesuré l'altération de la photosynthèse après infection n'ont que rarement quantifié cette dernière, ce qui restreint le nombre de comparaisons possibles. Owera et al. (1981) à propos de la rouille de l'orge indiquent une réduction de la photosynthèse inférieure à celle de la surface saine. Ils expliquent ce phénomène par un meilleur rendement de la chlorophylle chez les parties demeurées indemnes des feuilles contaminées. Le cas du $M$. brunnea apparait donc différent et original : nos observations montrent que la distorsion entre infection et photosynthèse nette est générale. Le bilan des composants de l'assimilation nette devient hétérogène selon les parties du limbe (nécroses, halos chlorotiques, tissus verts) sans qu'il soit possible actuellement d'en préciser l'ampleur, notamment à propos des tissus apparemment indemnes.

La comparaison des clones entre eux (tabl. 2) montre que rapportée à une surface infectée donnée, la perturbation de la photosynthèse augmente de "Robusta » au «Magister géant»ce qui est d'ailleurs conforme au classement traditionnel de ces clones. Un même degré d'infection n'implique donc pas le même dommage sur la physiologie de l'hôte selon son génotype. Lakso et al. (1982) avaient conclu de la même manière à propos de l'oïdium de la vigne. Les données relatives à la sporulation complètent cette comparaison des clones du point de vue épidémiologique. Ainsi à 50000 spores par $\mathrm{ml}$, la sporulation est nulle chez « Robusta », incomplète chez « I214 » et totale chez «Magister géant ». Toutes les taches sporulent lorsque la pression d'inoculum est forte mais dans le cas de "Robusta " la nécrose précoce des feuilles réduit leur caractère contagieux. Les approches physiologiques et épidémiologiques enrichissent ainsi la compréhension des différences de résistances entre cultivars et confortent le point de vue exprimé antérieurement par l'un d'entre nous (PINON, 1980) sur la multiplicité des modes d'expression de la résistance à $M$. brunnea .

\section{Conclusion, perspectives}

Les résultats présentés ci-dessus permettent d'éclairer d'un jour nouveau la physiologie des interactions entre $M$. brunnea et son hôte, en particulier en détectant les différences précoces de sensibilité entre clones qui échappent à l'analyse symptomatologique. La quantification de ces interactions sera poursuivie en faisant varier les pressions d'inoculum. 
Le rôle de la réduction de conductance stomatique dans la baisse de l'activité photosynthétique semble assez réduit. Une quantification plus précise à partir de modèles présentés par JoNEs (1985) sera engagée : de plus, l'effet réel de sensibilisation aux contraintes hydriques dû au dysfonctionnement stomatique dans les dégâts de la maladie sera analysé.

Cette étude a mis en relief des zones d'ombre dans nos connaissances sur le développement et l'expansion du mycélium dans les feuilles; une analyse simultanée de l'histologie des feuilles infectées et de leur physiologie (photosynthèse, respiration, photorespiration) devrait nous permettre de mieux comprendre les interactions en cause, et ce, à différents niveaux d'inoculation.

\title{
Remerciements
}

Les auteurs remercient Arlette Schipfer et Jean-Lu= Renoux de leur contribution technique, et Jean-Marc GueHL pour l'intérêt porté à ce travail.

Reçu le $1^{\text {er }}$ juillet 1986.

Accepté le 4 novembre 1986.

\author{
Summary \\ Photosynthesis of poplar leaves infected with M. brunnea : \\ compared evolution on three cultivars
}

\begin{abstract}
Marssonina brunnea, a fungal foliar parasite of cultivated poplars, induces small necrosis surrounded by a halo of chlorotic tissue. It is responsible for reductions in tree growth, first through reduced photosynthetic active leaf area and later by inducing early defoliation. Original data on the direct effect of the parasite on host's photosynthesis and stomatal conductance are presented here.
\end{abstract}

Three clones have been studied : a tolerant ( Robusta ), a susceptible («I-214») and a very susceptible one (« Magister géant »). Young shoots were inoculated with conidial solutions (50.000 and 100.000 conidia. $\mathrm{ml}^{-1}$ ) and gas exchange measured once daily under controlled conditions until fungal sporulation. Photosynthesis reduction was related at the end of the experiments to relative alterated leaf area (necrotic plus chlorotic tissues).

Two or three days after inoculation, at a moment where only epidermal cells are likely colonized by the fungus, net $\mathrm{CO}_{2}$ assimilation and stomatal conductance begin to decrease strongly. In a second step, approximatively at the time of leaf necrosis appearance, photosynthetic activity remains rather constant, even during fungal spirulation, or decreases only very slowly. Degree of alteration is strongly controlled by inoculum pressure and clone's susceptibility.

Alterated stomatal conductance seems to play a limited role in photosynthesis reduction: calculations of net assimilation at constant internal $\mathrm{CO}_{2}$ concentration show that the mesophyll conductance is strongly reduced. This reduction may be due to changes in respiratory balance, but this seems unlikely as $\mathrm{CO}_{2}$ compensation point remains; constant and fungal respiration is very limited. 
Compared with data on rusts and powdery mildew infection, $M$. brunnea exhibits a peculiar behaviour: reduction of net $\mathrm{CO}_{2}$ assimilation exceeds largely the extend which could be forecast by the extend of leaf area alterations.

The comparison of behaviour of the three clones highlights that tolerance to infection may be at least related to reduced sporulation ability of infecting fungus and to maintenance of a higher photosynthetic activity at a given level of infection.

Key words : Marssonina brunnea, Populus, photosynthesis, stomatal conductance, sensitivity.

\section{Références bibliographiques}

Anonyme, 1980. Peupliers et saules. FAO, Organisation des Nations Unies pour l'Alimentation et l'Agriculture, Rome, 343 p.

Ahmad J., Farrar J.F., Whitbread R., 1983. Photosynthesis and chloroplast functioning in leaves of barley infected with brown rust. Physiol. Plant Pathol., 23, 411-419.

Ayres P.G., 1980. Stomatal behaviour in mildewed pea leaves : solute potentials of the epidermis and effects of pisatin. Physiol. Plant Pathol., 17, 157-165.

Bousquet J.F., Skajennikoff M., Bethenod O., 1977. Action dépressive de l'ochracine, phytotoxine synthétisée par le Septoria nodorum (Berk.) Berk., sur l'assimilation du $\mathrm{CO}_{2}$ par des plantules de Blé. Ann. Phytopathol., 9, 503-510.

Cellerino G.P., Anselmi N., Pinon J., 1978. Influence de l'âge des feuilles de Peuplier sur la sensibilité à Marssonina brunnea. Eur. J. For. Path., 8, 273-279.

Cowan I.R., 1977. Stomatal behaviour and environment. Adv. Bot. Res., 4, 117-228.

Dickmann J.M., 1971. Photosynthesis and respiration by developing leaves of cottonwood (Populus deltoides Bartr.). Bot. Gaz., 132, 253-259.

Duniway J.M., Durbin R.D., 1971. Some effects of Uromyces phaseoli on the transpiration rate and stomatal response of bean leaves. Phytopathology, 61, 114-119.

Farquhar G.D., Sharkey T.D., 1982. Stomatal conductance and photosynthesis. Ann. Rev. Plant Physiol., 33, 317-345.

GaAstra P., 1959. Photosynthesis of crop plants as influenced by light carbon dioxide, temperature and stomatal diffusion resistance. Meded. Landbouwhogesch. Wageningen, 59, 1-68.

Gordon T.R., Duniway J.M., 1982 a. Effects of powdery mildew infection on the efficiency of $\mathrm{CO}_{2}$ fixation and light utilization by sugar beet leaves. Plant Physiol, 69, 139-142.

Gordon T.R., Duniway J.M., 1982 b. Stomatal behaviour and water relations in sugar beet leaves infected by Erysiphe polygoni. Phytopathology, 72, 723-726.

Hewitr H.G., Ayres P.G., 1975. Changes in $\mathrm{CO}_{2}$ and water vapour exchange rates in leave of Quercus robur infected by Microsphaera alphitoides (powdery mildew). Physiol. Plant Pathol., 7, $127-137$.

Jarvis P.G., 1971. The estimation of resistances to carbon dioxide transfer. In Plant Photosynthetic Production, Manual of Methods, ed. Z. Sestak, J. Catsky, P.G. Jarvis, pp. 566-631. The Hague : Junk, 818 p.

JoNES H.G., 1973. Limiting factors in photosynthesis. New Phytol, 72, 1089-1094.

Jones H.G., 1985. Partitioning stomatal and non-stomatal limitations to photosynthesis. Plant Cell. Environ., 8, 95-104

Lakso A.N., Prati C., Pearson R.C., Pool R.M., Sefm R.C., Walser M.J., 1982. Photosynthesis, transpiration, and water use efficiency of mature grape leaves infected with Uncinula necator (powdery mildew). Phytopathology, 72, 232-236.

LARSEN C.M., 1968. Le développement des stomates chez le genre Populus au cours de l'accroissement des feuilles. Silvae Genet., 17, 73-82. 
Maurer P., 1985. Interaction entre la photosynthèse des peupliers et leur infection par Marssonina brunnea (Ell. et Ev.) Magn. DAA protection des cultures, $29 \mathrm{p}$.

Maurer P., Pinon J., Genetej I., 1986. Méthode de mesure de la surface foliaire lésée par Marssonina brunnea (Ell. et Ev.) Magn. Ann. Sci. for., 43, 403-406.

Mignucci J.S., Boyer J.S., 1979. Inhibition of photosynthesis and transpiration in soybean infected by Microsphaera diffusa. Phytopathology, 69. 227-2.30.

Mitchell D.T., 1970. Carbon dioxide exchange by infected first leaf tissues susceptible to wheat stem rust. Trans. br. mycol. Soc., 72, 63-68.

OWfra S.A.P., Farrak J.F., Whitbread R., 1981. Growth and photosynthesis in barley infected with brown rust. Physiol. Plant Path., 18, 79-9i).

Pinon J., 1980. Etude des composants phénotypiques de la résistance du peuplier à Marssonina brunnea. XXI Conf. FAO/CIP/Dis.-IUFRO S2.05.1)3, Kornik, Poland (September 1-5), 141146.

Rowe J., REID J., 1979. Some aspects of carbon relations in the barley Helminthosporium teres complex. I. The effects of infection upon carboxylation in vivo and in vitro. Can. J. Bot., 57, 195-207

Scharen A.L.. Krupinsky J.M., 1969. Elfect of Septoria nodorum on $\mathrm{CO}_{2}$ absorption and yield of wheat. Phytopathology, 59, 1298-1301.

Spiers A.G., Hopcroft D.H., 1983. Ultrastructural study of the pathogenesis of Marssonina species to poplars. Eur. J. For. Path., 13. 414-427.

Walter D.R. AIRES P.G.. 1983. Changes in nitrogen and enzyme activities associated with $\mathrm{CO}$, exchanges in healthy leaves of powdery mildewinfected barley. Physiol. Plant Path., 23, 447459 . 